\title{
Asymptomatic moyamoya syndrome, atlantoaxial subluxation and basal ganglia calcification in a child with Down syndrome
}

\author{
Kyung Yeon Lee, MD', Kun-Soo Lee, MD², Young Cheol Weon, MD³ \\ ${ }^{1}$ Department of Pediatrics, Ulsan University Hospital, University of Ulsan College of Medicine, Ulsan, ${ }^{2}$ Department of Neurosurgery, Inje University Busan Paik \\ Hospital, Busan, ${ }^{3}$ Department of Radiology, Ulsan University Hospital, University of Ulsan College of Medicine, Ulsan, Korea
}

Down syndrome, the most common chromosomal abnormality, may be associated with various neurologic complications such as moyamoya syndrome, cervical spinal cord compression due to atlantoaxial subluxation, and basal ganglia damage, as well as epileptic seizures and stroke. Many cases of Down syndrome accompanied by isolated neurologic manifestations have been reported in children; however, Down syndrome with multiple neurologic conditions is rare. Here, we have reported a case of Down syndrome in a 10-year-old girl who presented with asymptomatic moyamoya syndrome, atlantoaxial subluxation with spinal cord compression, and basal ganglia calcification. To the best of our knowledge, this is the first report of Down syndrome, in a child, which was accompanied by these 3 neurologic complications simultaneously. As seen in this case, patients with Down syndrome may have neurologic conditions without any obvious neurologic symptoms; hence, patients with Down syndrome should be carefully examined for the presence of neurologic conditions.

Key words: Down syndrome, Moyamoya disease, Atlantoaxial instability, Basal ganglia calcification

\section{Introduction}

The patient with Down syndrome may present with diverse neurologic manifestations, including moyamoya syndrome, cervical spinal cord compression related to atlantoaxial subluxation, and basal ganglia damage ${ }^{1-4)}$. Although there are many cases of children with Down syndrome accompanied by isolated aforementioned neurologic manifestations, it is rare that children with Down syndrome have multiple neurologic problems, simultaneously. We describe a case of a child with Down syndrome, who had asymptomatic Moyamoya syndrome, atlantoaxial subluxation, and basal ganglia calcification, simultaneously.

\section{Case report}

A 10-year-old girl with Down syndrome was referred to the pediatric department from the ophthalmologic department of Ulsan University Hospital because both basal ganglia calcification was incidentally detected on a brain computed tomography (Fig. 1). She had been managed at the ophthalmologic clinic because of strabismus. Brain computed tomography (CT) was performed to identify whether her ophthalmologic manifestations, including esotropia of both eyes, amblyopia in the left eye, cyclotorsion of both eyes, and
Corresponding author: Kyung Yeon Lee, MD Department of Pediatrics, Ulsan University Hospital, University of Ulsan College of Medicine, 877 Bangeojinsunhwan-doro, Dong-gu, Ulsan 682-714, Korea

Tel: $+82-52-250-8980$

Fax: +82-52-250-8071

E-mail: pdsnoopy@naver.com

Received: 23 August 2012

Revised: 29 November 2012

Accepted: 9 May 2013
Copyright (C) 2013 by The Korean Pediatric Society

This is an open-access article distributed under the terms of the Creative Commons Attribution NonCommercial License (http://creativecommons.org/ licenses/by-nc/3.0/) which permits unrestricted noncommercial use, distribution, and reproduction in any medium, provided the original work is properly cited. 
horizontal nystagmus, would result from brain parenchymal lesion.

She was born at Ulsan University Hospital. At that time, she showed typical features of Down syndrome, and was diagnosed with Down syndrome by the identification of $47, \mathrm{XX},+21$ on the cytogenetic study. Echocardiography after birth, which was performed due to cardiac murmur, revealed a $6.3 \mathrm{~mm}$ sized perimembranous ventricular septal defect and two small sized atrial septal defects. She had been examined by regular follow-up echocardiography evaluation without surgery, and

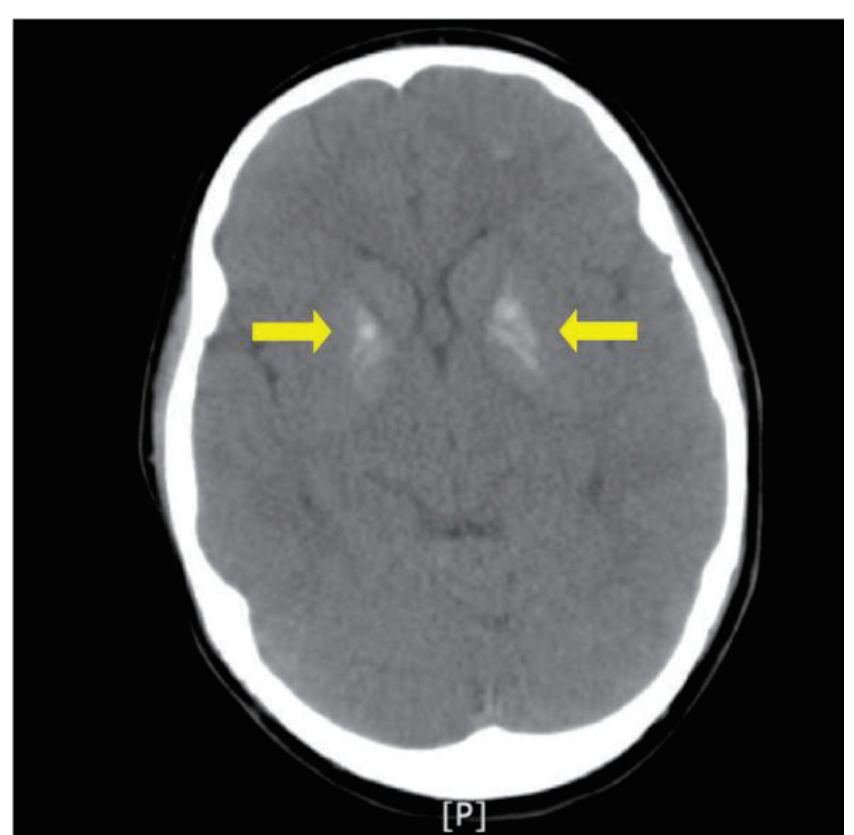

Fig. 1. Brain computed tomography shows calcifications in the bilateral basal ganglia (arrow). was presented with no symptoms associated with cardiac anomalies until the time of admission. She had moderate mental retardation with full scale intelligence quotient score of 44 . Her parents stated that they had never noticed apparent neurologic symptoms of the patient, including transient motor weakness before the admission.

On physical examination at the time of admission, she showed typical features of Down syndrome, including hypertelorism, depressed nasal bridge, low-set ears, epicanthal folds, protruded tongue, small chin, and simian creases on both hands. On cardiac auscultation, systolic murmur was heard. On neurologic examination, she was fully alert and conscious. She showed esotropia of both eyes, but, the limitation of both eye movements was absent. She exhibited horizontal nystagmus, without vertical nystagmus. No obvious other abnormalities were revealed on the cranial nerve examination. Motor weakness of four extremities was absent, although muscle tone was slightly hypotonic. The deep tendon reflexes on both knees were slightly exaggerated, and Babinski sign was absent in both. On laboratory findings, we observed total calcium, $9.8 \mathrm{mg} / \mathrm{dL}$; ionized calcium, 4.60 $\mathrm{mg} / \mathrm{dL}$; parathyroid hormone, $44.94 \mathrm{pg} / \mathrm{mL}$; ceruloplasmin, 22 $\mathrm{mg} / \mathrm{dL}$; serum copper, $96 \mu \mathrm{g} / \mathrm{dL}$; thyroid-stimulating hormone, $1.20 \mathrm{mIU} / \mathrm{L}$; and free thyroxine, $1.49 \mathrm{ng} / \mathrm{dL}$. Investigations for autoimmune (antinuclear antibody, antidouble strand DNA antibody, anticardiolipin antibodies, antiphospholipid antibodies, and antineutrophilic cytoplasmic antibody), prothrombotic (protein C, S, Factor V Leiden mutation, and antithrombin III) and metabolic (serum amino acid and urine organic acid) disorders were within normal limits. Echocardiography performed, during the admission, revealed 3-mm sized perimembranous ventricular septal defect with small to moderate left to right shunt, but good ventricular function. Brain magnetic resonance imaging and

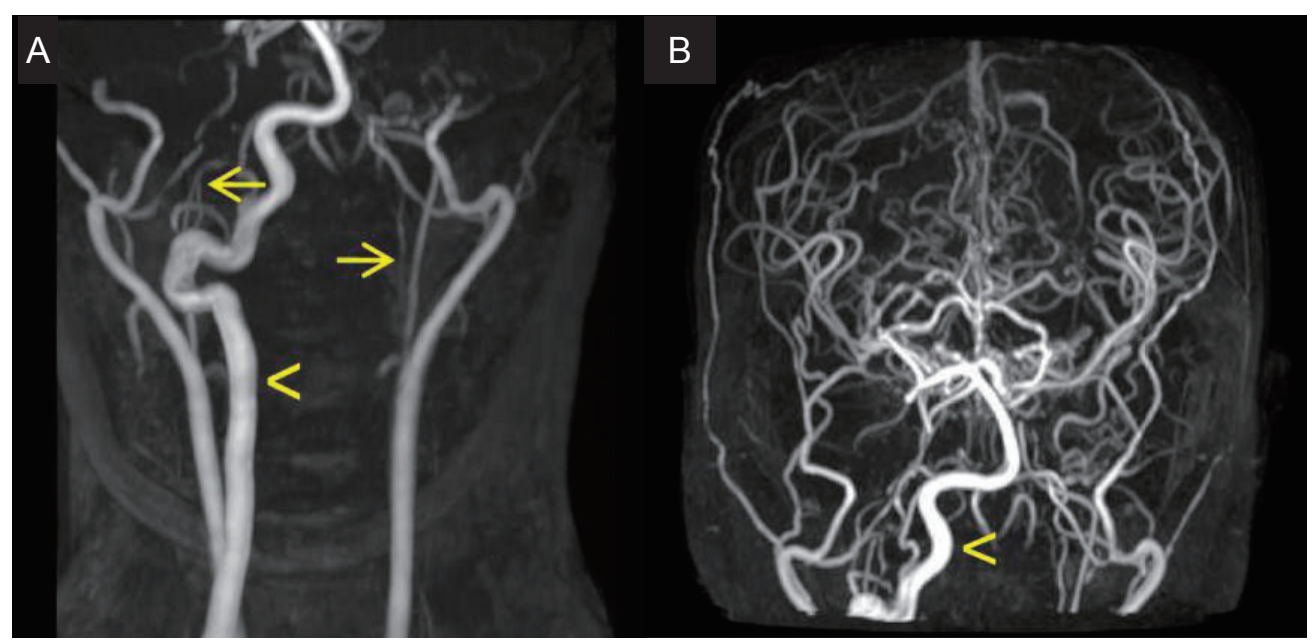

Fig. 2. Time of flight magnetic resonance angiography. (A) Severe stenosis of the bilateral internal carotid arteries (arrows) is observed. (B) A hypertrophic right vertebral artery (arrowhead) supplies collateral blood flow to the bilateral cerebral hemispheres. 
magnetic resonance angiography, which were performed during the admission, showed as follows; multiple tiny ischemic lesions in both frontal white matters, compression of the cervical cord due to displaced odontoid process at the level of $\mathrm{C} 1$, and severe stenosis of the bilateral internal carotid arteries with collaterals to the bilateral cerebral hemispheres (Figs. 2, 3A). Four vessels angiography revealed occlusion of the proximal portion of the right internal carotid artery, occlusion of the left internal carotid artery bifurcation and the left middle cerebral artery, transdural collaterals from the middle meningeal, the superficial temporal, and the occipital arteries, and hypertrophic right vertebral artery with prominent leptomeningeal collaterals to both cerebral hemispheres. Three dimensional cervical spine CT showed subluxation and rotation of $\mathrm{C} 2$ on $\mathrm{C} 1$ with spinal canal narrowing, and os odontoideum (Fig. 3B).

Her family moved to another province far from our hospital, several months after the evaluation. According to the telephone interview with the patient's mother, the patient had been doing well with no neurologic symptom and no special management during the 2 years since the admission to our hospital.

\section{Discussion}

Down syndrome may be associated with various neurologic complications, including moyamoya syndrome, cervical spinal cord compression related to atlantoaxial subluxation, and basal ganglia damage, as well as epileptic seizures, stroke, and
Alzheimer disease ${ }^{1-4)}$.

Moyamoya syndrome has been reported to occur with a higher frequency in Down syndrome than in the general pediatric population ${ }^{5)}$. Moyamoya syndrome is a cerebrovascular disorder, characterized by a slowly progressive bilateral stenosis, eventual occlusion of the supraclinoid internal carotid arteries and produces collateral vessels. It is different from moyamoya disease in that moyamoya syndrome occurs secondary to a variety of slow progressive, occlusive cerebral vasculopathies and genetic syndromes, such as sickle cell disease, postradiation vasculopathy, Down syndrome, neurofibromatosis, and Williams syndrome ${ }^{2)}$. The exact mechanism of vascular occlusion in Down syndrome with moyamoya syndrome is unknown, although several hypotheses have been proposed ${ }^{6-11)}$. Patients with Down syndrome are generally predisposed to vascular abnormalities, such as abnormal nailbed capillary morphology, high pulmonary vascular resistance with congenital heart disease, abnormalities of retinal vessels and primary intimal fibroplasias ${ }^{6}$. In respect of the molecular basis, several proteins associated with the increased risk for vascular disease, such as alpha chains of collagen type VI, superoxide dismutase I, interferon gamma receptor and cystathionine beta synthase, are encoded on chromosome $21^{6-9)}$. Autoimmunity and protein $\mathrm{C}$ deficiency has also been postulated as other possible mechanisms associated with Down syndrome and moyamoya syndrome ${ }^{10,11)}$. In our case, however, the work-ups for autoimmune and hypercoagulation disorders were all negative.

Atlantoaxial instability occurs in $10 \%$ to $30 \%$ of patients with
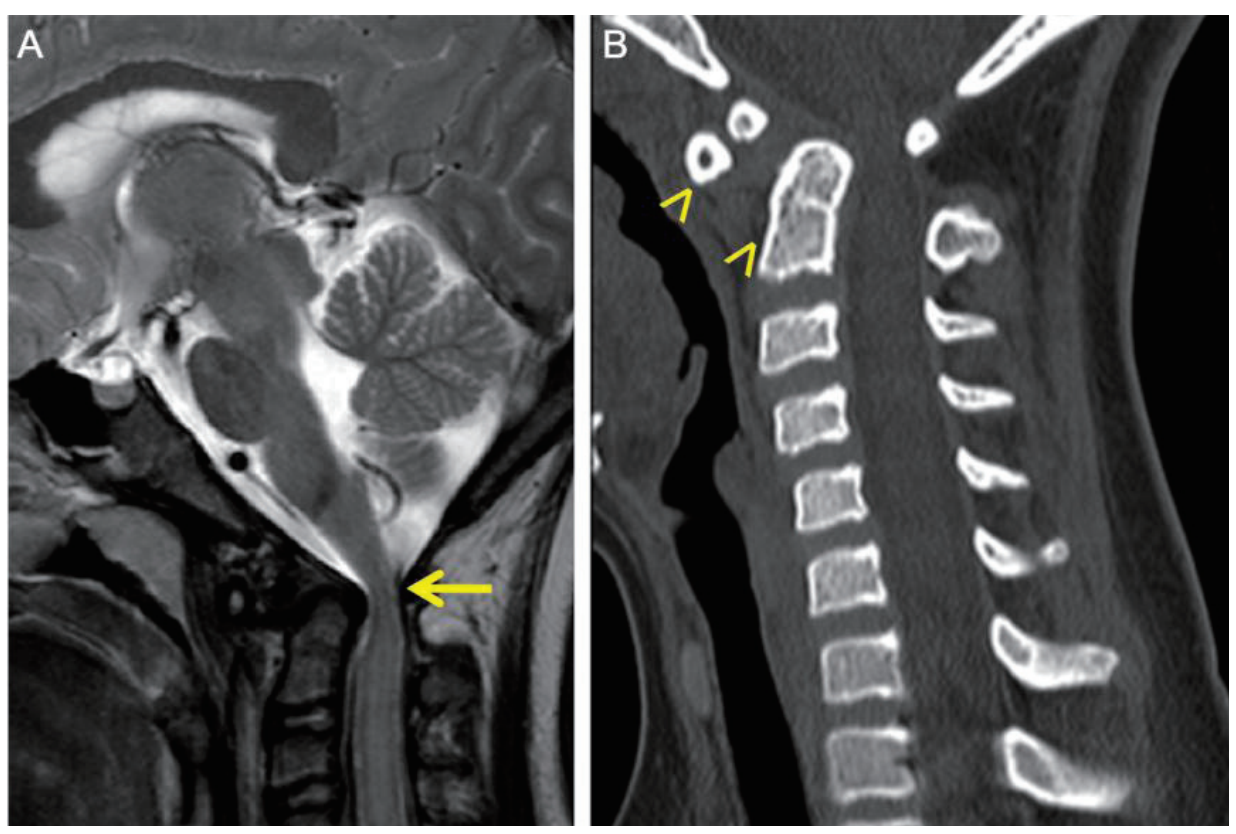

Fig. 3. (A) T2-weighted sagittal image shows compression of the cervical cord (arrow), due to the displaced odontoid process at the level of $\mathrm{C} 1$. (B) Cervical spinal computed tomography scan shows subluxation of $\mathrm{C} 2$ with os odontoideum (arrowheads). 
Down syndrome, the majority of which are asymptomatic, with estimates of symptomatic disease ranging from $1 \%$ to $2 \%{ }^{12)}$. Atlantoaxial instability in the Down syndrome population may be secondary to bony anomalies, such as os odontoideum, which occurs in approximately 6\% of children with Down syndrome ${ }^{13)}$. Other proposed causes for atlantoaxial instability in Down syndrome include intrinsic collagen defects that lead to transverse ligament laxity, and a chronic inflammatory state that weakens the ligamentous structures ${ }^{14,15}$. Atlantoaxial subluxation narrows the anteroposterior diameter of the neural canal, potentially compressing the spinal cord at the level of C1. It is generally accepted that ligamentous instability at the atlantoaxial joint in patients with Down syndrome is unlikely to progress to clinically relevant subluxation ${ }^{12)}$. However, those children with bony anomalies are at higher risk of progression and should be followed closely. In our case, although obvious neurologic manifestations associated with moyamoya syndrome and atlantoaxial subluxation did not appear, it should be kept in mind that neurologic symptoms may develop later, considering her age and the progressive characteristics of moyamoya syndrome and os odontoideum.

Incidence of basal ganglia calcification, in patients with Down syndrome, is very high compared to the general population (10$45 \%$ vs. $0.3-0.6 \%)^{16}$. Basal ganglia calcification in the normal population is occasionally observed in older patients above 40 years of age. In Down syndrome, basal ganglia calcification appears at a younger age, which perhaps indicates premature aging ${ }^{17)}$. The pathogenesis of basal ganglia calcification in Down syndrome is unknown. Takashima and Becker ${ }^{16)}$ found basal ganglia calcification was localized to a constant area of globus pallidus, calcification was increasingly severe with aging and calcification and amyloid degeneration of the adjacent blood vessels were present. They suggested that progressive structural change occurs in the vessel walls with advancing age in patients with Down syndrome. Basal ganglia calcification in Down syndrome is usually asymptomatic, and is detected incidentally by neuroimaging, although some reports associated with neurologic disorders, such as psychosis, movement disorder, have been documented ${ }^{18,19)}$.

To the best of our knowledge, this is the first report that a child with Down syndrome had moyamoya syndrome, atlantoaxial subluxation, and basal ganglia calcification, simultaneously. Because patients with Down syndrome may have multiple neurologic problems simultaneously, even without obvious neurologic symptoms like our case, judicious practice will be necessary in the evaluations for patients with Down syndrome.

\section{Conflict of interest}

No potential conflict of interest relevant to this article was reported.

\section{References}

1. Larner AJ. Down syndrome in the neurology clinic: Too much? Too little? Too late? Downs Syndr Res Pract 2007;12:69-71.

2. Deveber GA. Cerebrovascular disease. In: Swaiman KF, Ashwal S, Ferriero DM, editors. Pediatric neurology: principles \& practice. 4th ed. Philadelphia: Mosby Elsevier, 2006:1759-95.

3. Ham JH, Song YK, Kim JI, Choi JK, Lee JY. Moyamoya syndrome in a 42-year-old man with Down syndrome. J Korean Neurol Assoc 2012;30:230-1.

4. Kim SY, Kim CY. A study on the atlantoaxial instability in children with Down syndrome. J Korean Pediatr Soc 1993;36:179-84.

5. Fukushima Y, Kondo Y, Kuroki Y, Miyake S, Iwamoto H, Sekido $\mathrm{K}$, et al. Are Down syndrome patients predisposed to Moyamoya disease? Eur J Pediatr 1986;144:516-7.

6. Dai AI, Shaikh ZA, Cohen ME. Early-onset Moyamoya syndrome in a patient with Down syndrome: case report and review of the literature. J Child Neurol 2000;15:696-9.

7. Korenberg JR. Toward a molecular understanding of Down syndrome. Prog Clin Biol Res 1993;384:87-115.

8. Nordstrom M, Kjellstrom T. Age dependency of cystathionine betasynthase activity in human fibroblasts in homocyst(e)inemia and atherosclerotic vascular disease. Atherosclerosis 1992;94:213-21.

9. Hansson GK, Holm J. Interferon-gamma inhibits arterial stenosis after injury. Circulation 1991;84:1266-72.

10. Leno C, Mateo I, Cid C, Berciano J, Sedano C. Autoimmunity in Down's syndrome: another possible mechanism of Moyamoya disease. Stroke 1998;29:868-9.

11. Gururaj A, Hardy D, Al-Gazali LI, Sztriha L, Roos A, Nork M. Are the strokes in moyamoya syndrome associated with Down syndrome due to protein C deficiency? Brain Dev 2002;24:719-22.

12. Hankinson TC, Anderson RC. Craniovertebral junction abnormalities in Down syndrome. Neurosurgery 2010;66(3 Suppl):32-8.

13. Semine AA, Ertel AN, Goldberg MJ, Bull MJ. Cervical-spine instability in children with Down syndrome (trisomy 21). J Bone Joint Surg Am 1978;60:649-52.

14. Pueschel SM, Scola FH. Atlantoaxial instability in individuals with Down syndrome: epidemiologic, radiographic, and clinical studies. Pediatrics 1987;80:555-60.

15. Tassone JC, Duey-Holtz A. Spine concerns in the Special Olympian with Down syndrome. Sports Med Arthrosc 2008;16:55-60.

16. Takashima S, Becker LE. Basal ganglia calcification in Down's syndrome. J Neurol Neurosurg Psychiatry 1985;48:61-4.

17. Wisniewski KE, French JH, Rosen JF, Kozlowski PB, Tenner M, Wisniewski HM. Basal ganglia calcification (BGC) in Down's syndrome (DS): another manifestation of premature aging. Ann N Y Acad Sci 1982;396:179-89.

18. Thase ME. Basal ganglia calcification and psychosis in Down's syndrome. Postgrad Med J 1984;60:137-9.

19. Okano S, Takeuchi Y, Kohmura E, Yoshioka H, Sawada T. Globus pallidus calcification in Down syndrome with progressive neurologic deficits. Pediatr Neurol 1992;8:72-4. 